\title{
Begünstigen psychosoziale Faktoren diabetische Fußulzera?
}

\author{
Inwieweit unterscheiden sich Pati- \\ enten mit diabetischen Fußulzera bei \\ verschiedenen psychosozialen Ver- \\ haltensweisen, besonders bezüglich \\ mentaler Störungen und selbstschä- \\ digendem Verhalten?
}

- Außer den physiologischen Ursachen (periphere Polyneuropathie, pAVK und Infektionen) für diabetische Fußulzerationen, gibt es viele Verhaltensweisen, die das Auftreten von Ulzera begünstigen. So sind eine gute Diabeteseinstellung, eine regelmäßige Fußpflege und das Tragen von protektivem Schuhwerk sicher günstig, um Ulzera zu vermeiden. Diese Verhaltensweisen sind wesentlich geprägt durch verschiedene psychosoziale Faktoren.

Insgesamt gibt es bisher nur wenige Studien zu psychosozialen Faktoren bei der Pathogenese von diabetischen Fußulzera. Vor allem gab es bisher keine Studien zu selbstschädigendem Verhalten bei Patienten mit diabetischen Fußulzerationen.

In dieser Querschnittsstudie untersuchte man zwischen 2006 und 2008 136 Patienten mit Typ-2-Diabetes, die in der Universität Dresden betreut wurden. Es gab zwei Gruppen mit je 47 Patienten, die entweder ein Fußulkus hatten oder nicht. Bezüglich des Alters, des Geschlechts und der Diabetesdauer waren diese beiden Gruppen gematcht.

Alle Patienten wurden mit dem standardisierten „Composite International Diagnostic Interview" befragt, um mentale Störungen zu erheben. Hierbei wurde auch der Alkohol- und Tabakkonsum abgefragt. Außerdem wurden andere selbstschädigende Verhaltensweisen mit dem „Assessment Scale of Self-Destruction" dokumentiert. Zusätzlich wurden weitere Fragebogen angewendet.

Wie üblich bei Patienten mit diabetischem Fußsyndrom, überwog der Anteil der Männer mit 74\%. Die Patienten mit Fußulzera hatten signifikant häu-

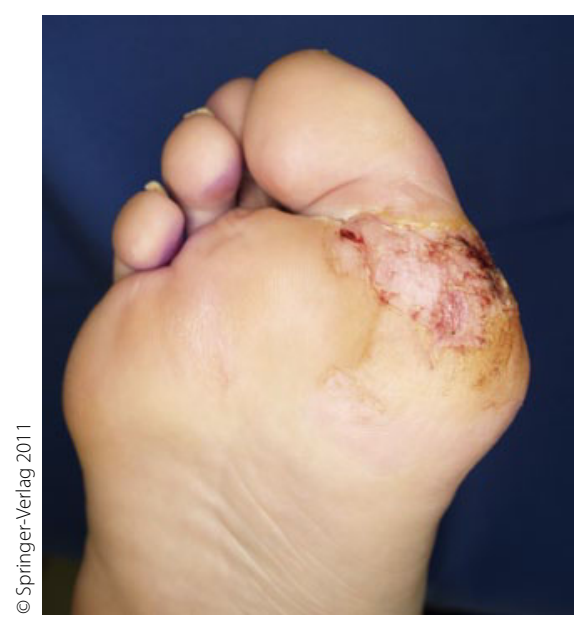

Patienten mit Fußulzera trinken mehr und verdienen weniger als solche ohne.

figer eine periphere diabetische Polyneuropathie. Das mittlere Netto-Einkommen der Ulkuspatienten war signifikant niedriger mit $1189 €$ versus $1535 €$ bei den Patienten ohne Fußulkus. Auch die Schulausbildung war bei den Patienten mit Ulzera um zwei Jahre kürzer.

Hinsichtlich der psychosozialen Faktoren fanden sich keine Unterschiede zwischen den beiden Gruppen bei depressiven Symptomen, affektiven Störungen, und der mentalen Lebensqualität. Unterschiede bestanden hingegen bezüglich einer niedrigeren physischen Lebensqualität und einer geringeren Neigung zu Angststörungen während der letzten zwölf Monate. Die Patienten mit Fußulzera suchten in den letzten zwölf Monaten auch weniger psychologische Betreuung und hatten weniger Psychopharmaka verschrieben bekommen. Es fand sich bei ihnen auch ein Trend zu weniger Arztbesuchen und weniger Arzneimitteltherapie insgesamt.

Beide Gruppen unterschieden sich nicht bezüglich des Ausmaßes des Übergewichts und des Tabakkonsums. Deutliche Unterschiede fanden sich jedoch beim Alkoholkonsum, der in der Gruppe der Patienten mit Fußulzera signifi- kant höher lag, mit 43\% Alkoholabhängigkeit im Vergleich zu 19\% in der Kontrollgruppe. Andere Arten von selbstschädigendem Verhalten unterschieden sich nicht signifikant zwischen beiden Gruppen.

Die Autoren folgern aus ihren Daten, dass ihre Patienten mit Fußulzera einen niedrigeren sozialen Status und niedrige Grade für Ängste hatten, weniger externe Unterstützung in Anspruch nahmen sowie eine passive Konfliktlösungsstrategie mit erhöhtem Alkoholkonsum aufwiesen.

\section{Kommentar}

Die Einsichtsfähigkeit und Mitarbeit der Patienten mit diabetischem Fußsyndrom, insbesondere bezüglich einer konsequenten Entlastung bei gefühllosem Fuß, ist häufig unzureichend. Ein auffälliges psychosoziales Verhalten bei einer Reihe von Patienten mit neuropathischem Fußsyndrom wird immer wieder deutlich, bisher gab es jedoch nur wenige Studiendaten hierzu. Ob das psychosoziale Muster, das in dieser relativ kleinen explorativen Querschnittsstudie gefunden wurde, wirklich typisch ist für Patienten mit diabetischem Fußsyndrom, muss in größeren prospektiven Studien gesichert werden.

M. SPRAUL

- N. Altenburg et al.

Alcohol consumption and other psycho-social conditions as important factors in the development of diabetic foot ulcers. Diabet. Med. 28 (2011) 168-174 